\title{
Opportunities for recommended mental health strategies to reduce stress at work
}

\author{
Robin De Croon, Francisco Gutiérrez \\ and Katrien Verbert
}

KU Leuven, Department of Computer Science, Celestijnenlaan 200A, BE-3001 Leuven, Belgium robin.decroon@kuleuven.be, francisco. gutierrez@kuleuven.be, katrien.verbert@kuleuven.be

\begin{abstract}
Human resource management is undergoing a profound change in digital transformation: HR departments are expected to build a compelling employee experience and redesign entire talent practices. Achieving this requires tackling the mental health problems at both individual and workplace levels including proactively managing mental health of the employees. To meet such demanding transformations, interactive technologies to mitigate workplace related risk factors could offer a huge potential. However, they are currently underexplored. In this position paper, we explore four research themes that could be addressed in a research agenda in the area of user experiences and wellbeing at work: 1) personalized, in-time stress management therapies, 2) the application of health recommender systems to tailor interventions to employees, 3) the involvement of professional coaches to enable employees to explore recommendations outside therapy sessions, and 4) personalized gamification mechanics to increase employees' adherence to both recommended therapies and tracking data.
\end{abstract}

\section{Keywords}

Stress $\cdot$ mental well-being $\cdot$ mHealth $\cdot$ adherence $\cdot$ gamification

How to cite this book chapter:

De Croon, R., Gutiérrez, F. and Verbert, K. 2020. Opportunities for recommended mental health strategies to reduce stress at work. In: Loizides, F., Winckler, M., Chatterjee, U., Abdelnour-Nocera, J. and Parmaxi, A. (eds.) Human Computer Interaction and Emerging Technologies: Adjunct Proceedings from the INTERACT 2019 Workshops. Pp. 157-162. Cardiff: Cardiff University Press. DOI: https://doi. org/10.18573/book3.t. License: CC-BY 4.0. 


\section{Introduction}

In 2015, 24.4\% of EU employees were experiencing frequent or constant stress at work and $35.2 \%$ felt exhausted [18]. Besides the occupational burnout, longterm stress at work can cause multiple mental health disorders, such as anxiety, depression, chronic widespread pain, and concentration difficulties. Workinduced stress can be responsible for a large employee turnover/retirement and is often associated with an increased risk of depressive symptoms and clinical depression among employees [16]. Studies estimate that around 50\% of all lost working days have some relation to work stress [4]. The cost to Europe of workrelated depression was recently estimated to be 617 billion euros annually [17].

Although the abundance of sensors and tracking technologies allow development of tailored and personalized services to improve work satisfaction, productivity, and health of employees, research into assessing mental conditions is currently mainly conducted in theoretical studies. Real life uses in a professional context require additional researched methods, that are highly adaptive to differences in employee personalities and workplace settings. In this position paper, we explore opportunities for research into solutions tailored to the individual employee; into solutions that provide data-driven, actionable insights, but also into those solutions that are supervised and tailored by a professional coach. On the other hand, such remote solutions typically suffer from low adherence. It should, therefore, be researched how personalized gamification mechanics can be used in the context of well-being at work.

\section{Personalized, in-time stress management therapies}

Several studies have gathered positive impact on the use of mobile and/or online therapies for the treatment of health problems related to stress and anxiety $[14,22]$. After all, mobile therapies are accessible $24 / 7$ and can be customized depending on user needs and may reduce barriers to face-to-face help-seeking, such as the stigma or discomfort about discussing one's own mental health [13]. In the past few years, a large number of mobile applications were developed to help prevent and treat mental problems, e.g., to reduce stress and behavior disorders or to improve cognitive functions. However, they are mainly low-value apps, often lack scientific evidence $[9,11]$, and are largely knowledge-based in the way that they guide the user towards respected sources of information [19]. Despite a large number of apps available, the evidence base is still scarce and needs additional research.

A 2013 review of mobile mental health apps identified eight papers describing only five apps [9]. Four of the five apps demonstrated significant reductions in depression, stress, and substance use, although a number of issues with the quality of these studies suggest these conclusions need to be interpreted cautiously. A 
more recent systematic review [11] in 2017 included 24 publications, of which 15 described an app. They concluded that there is currently insufficient research evidence to support the effectiveness of mental health apps. "Given the number and pace at which mHealth apps are being released on app stores, methodologically robust research studies evaluating their safety, efficacy, and effectiveness is promptly needed" [11]. Furthermore, another high-quality systematic review [1] performed in 2016 concluded that mental health apps are not personalized towards the end-user, which provides an important research opportunity as Bakker et al. [1] stress that "[t]ailored interventions are more efficacious than is rigid self-help."

\section{Health recommender systems to tailor interventions}

In recent years, there has been an increasing interest to apply existing recommender techniques in a variety of health-related apps to provide actionable suggestions to end-users [20-21]. The overall objective is to empower people to monitor and improve their health with technology-assisted coaching through personalized recommendations. However, there is currently little research on health recommenders which provide stress related insights, especially in a professional context. For example, recommenders to alleviate stress include recommending books to read [23] or meditative audios [24]. The most relevant study is the RCT of Bidargaddi et al. [2] where actual patients (not regular employees, but people with a known health condition) were enrolled. A large group of patients $(\mathrm{n}=192+195$ control) were asked to use a web-based recommendation service for four weeks that recommended mental health and well being mobile applications. Their self-guided app recommendation service was able to halt a decline in mood, energy, and sleep.

\section{Involvement of coaches}

A third important topic for a research agenda is a stronger involvement of professional coaches. Similar to concordance, an "alternative model proposed by the medical field that favours an equal and collaborative patient-doctor relationship in the negotiation of care" [12], a professional coach and an employee should develop a relationship that involves a collaborative empiricism [5]; a collaboration to motivate employees effectively. This will enable employees to explore recommended strategies and beliefs outside their therapy session. This would ideally involve encouraging users to develop their own hypotheses about what may happen as a result of using the app or participating in certain activities. An active collaboration can thus support autonomy and provide opportunities in a company for the development of competence in behavioral, emotional, or cognitive self-management. 


\section{Gamification to increase adherence}

The lack of adherence, "the extent to which individuals experience the content to derive maximum benefit from the intervention, as defined or implied by its creators" [15], to remote solutions is well documented [10]. However, little research can be found on how and what principles to implement in a workplace context. We, therefore, argue that researching how to increase employees' adherence to mental health therapies is crucial in a new research agenda. One potential approach to increase employees' experience is the use of gamification mechanics, i.e., "the use of game design elements in non-game contexts" [8]. After all, gamification harnesses the motivational affordances of gameful experiences to influence psychological outcomes and further behavioral outcomes and already has multiple applications in the healthcare domain [6].

Effective gamification is a combination of game design, behavioral economics, motivational psychology, and user experience and user interface design [3]. If implemented well, gamification can increase the motivation of user's trajectories [7]. However, gamification is not a panacea [6]. Different user groups and personality types necessitate different gamification mechanics. Hence, there is a need for research with respect to which gamification mechanics are appropriate for specific personality types and user groups. Careful consideration is needed to select a set of gamification mechanics for a specific user group. Finally, it is less known how gamification can be used within a sensitive setting such as the workplace where employees submit private health data.

\section{Conclusion and future work}

In this position paper, we proposed our position, that there is a demand and utility for a personalized and in-time stress management platform in a workplace context. By conducting a brief literature research, we came to the reasoned opinion, that the combination of health recommender systems, the involvement of professional coaches, and the use of gamification might have the potential to mitigate work-induced stress and thus increase the user experience and wellbeing of employees in a workplace context. However, this claim needs stronger research foundation and should be addressed in future research.

\section{Acknowledgements}

This work is part of the research project Personal Health Empowerment with project number HBC.2018.2012 and Panacea project with project number HBC.2016.0177, which are financed by Flanders Innovation \& Entrepreneurship. 


\section{References}

1. Bakker, D., Kazantzis, N., Rickwood, D., Rickard, N.: Mental health smartphoneapps: review and evidence-based recommendations for future developments. JMIR mental health 3(1), e7 (2016)

2. Bidargaddi, N., Musiat, P., Winsall, M., Vogl, G., Blake, V., Quinn, S., Orlowski, S., Antezana, G., Schrader, G.: Efficacy of a web-based guided recommendation service for a curated list of readily available mental health and well-being mobile apps for young people: Randomized controlled trial. J Med Internet Res 19(5), e141 (May 2017)

3. Chou, Y.k.: Actionable gamification: Beyond points, badges, and leaderboards. Octalysis Group (2015)

4. Cox T, Griffiths A, R.G.E..: Research on Work-related Stress. Tech. rep., Officefor Official Publications of the European Communities, Luxembourg (2000)

5. Dattilio, F.M., Hanna, M.A.: Collaboration in cognitive-behavioral therapy.Journal of Clinical Psychology 68(2), 146-158 (2012)

6. De Croon, R., Wildemeersch, D., Wille, J., Verbert, K., Vanden Abeele, V.: Gamification and serious games in a healthcare informatics context. In: IEEE International Conference on Healthcare Informatics. pp. 53-63. IEEE (2018)

7. De Schutter, B., Vanden Abeele, V.: Designing meaningful play within the psychosocial context of older adults. In: Proceedings of the 3rd International Conference on Fun and Games. pp. 84-93. ACM (2010)

8. Deterding, S., Dixon, D., Khaled, R., Nacke, L.: From game design elementsto gamefulness: defining gamification. In: Proceedings of the 15th International Academic MindTrek Conference: Envisioning Future Media Environments. pp. 9-15. ACM (2011)

9. Donker, T., Petrie, K., Proudfoot, J., Clarke, J., Birch, M.R., Christensen, H.: Smartphones for smarter delivery of mental health programs: A systematic review. J Med Internet Res 15(11), e247 (Nov 2013)

10. Eysenbach, G.: The law of attrition. Journal of medical Internet research 7(1), e11 (2005)

11. Grist, R., Porter, J., Stallard, P.: Mental health mobile apps for preadolescentsand adolescents: a systematic review. Journal of medical internet research 19(5), e176 (2017)

12. Grönvall, E., Verdezoto, N., Bagalkot, N., Sokoler, T.: Concordance: A criticalparticipatory alternative in healthcare it. Aarhus Series on Human Centered Computing 1(1), 4 (Oct 2015)

13. Gulliver, A., Griffiths, K.M., Christensen, H.: Perceived barriers and facilitators tomental health help-seeking in young people: a systematic review. BMC psychiatry 10(1), 113 (2010)

14. Heber, E., Ebert, D.D., Lehr, D., Cuijpers, P., Berking, M., Nobis, S., Riper, H.: The benefit of web-and computer-based interventions for stress: a 
systematic review and meta-analysis. Journal of medical Internet research 19(2), e32 (2017)

15. Kelders, S.M., Kok, R.N., Ossebaard, H.C., Van Gemert-Pijnen, J.E.: Persuasivesystem design does matter: a systematic review of adherence to web-based interventions. Journal of medical Internet research 14(6), e152 (2012)

16. Madsen, I.E., Nyberg, S.T., Hanson, L.M., Ferrie, J.E., Ahola, K., Alfredsson,L., Batty, G.D., Bjorner, J.B., Borritz, M., Burr, H., et al.: Job strain as a risk factor for clinical depression: systematic review and meta-analysis with additional individual participant data. Psychological medicine 47(8), 1342-1356 (2017)

17. Matrix: Economic analysis of workplace mental health promotion and mental disorder prevention programmes and of their potential contribution to EU health, social and economic policy objectives. Tech. Rep. May (2013)

18. Parent-Thirion, A., Biletta, I., Cabrita, J., Vargas, O., Vermeylen, G., Wilczynska,A., Wilkens, M.: 6th European Working Conditions Survey: Overview Report. Eurofound (Europ. Foundation for the Improvement of Living and Working (2016)

19. Payne, H.E., Wilkinson, J., West, J.H., Bernhardt, J.M.: A content analysis of precede proceed constructs in stress management mobile apps. Mhealth 2 (2016)

20. Rabbi, M., Aung, M.H., Zhang, M., Choudhury, T.: MyBehavior: automatic personalized health feedback from user behaviors and preferences using smartphones. In Proceedings of the 2015 ACM International Joint Conference on Pervasive and Ubiquitous Computing (UbiComp '15) pp. 707-718 (2015)

21. Radha, M., Willemsen, M.C., Boerhof, M., IJsselsteijn, W.A.: Lifestyle recommendations for hypertension through rasch-based feasibility modeling. In: Proceedings of the 2016 Conference on User Modeling Adaptation and Personalization. pp. 239-247. UMAP '16, ACM, New York, NY, USA (2016)

22. Thórarinsdóttir, H., Kessing, L.V., Faurholt-Jepsen, M.: Smartphone-based selfassessment of stress in healthy adult individuals: a systematic review. Journal of medical Internet research 19(2), e41 (2017)

23. Xin, Y., Chen, Y., Jin, L., Cai, Y., Feng, L.: TeenRead: An Adolescents Reading Recommendation System Towards Online Bibliotherapy. Proceedings - 2017 IEEE 6th International Congress on Big Data, BigData Congress 2017 pp. 431-434 (2017)

24. Zaini, N., Latip, M.F.A., Omar, H., Mazalan, L., Norhazman, H.: Onlinepersonalized audio therapy recommender based on community ratings. ISCAIE 2012 - 2012 IEEE Symposium on Computer Applications and Industrial Electronics pp. 318-322 (2012) 